\title{
The anomalous $X$-ray pulsar 1E 1048.1-5937: Phase resolved spectroscopy with the XMM-Newton satellite
}

\author{
A. Tiengo ${ }^{1,2}$, E. Göhler ${ }^{3}$, R. Staubert ${ }^{3}$, and S. Mereghetti ${ }^{1}$ \\ 1 Istituto di Fisica Cosmica "G. Occhialini", via Bassini 15, 20133 Milano, Italy \\ 2 XMM-Newton Science Operation Center, Vilspa ESA, Apartado 50727, 28080 Madrid, Spain \\ 3 Univ. Tübingen, Inst. fur Astronomie und Astrophysik, Abt. Astronomie, Waldhauserstr., 72076 Tübingen, \\ Germany
}

Received 18 September 2001 / Accepted 21 November 2001

\begin{abstract}
We report on an observation of the anomalous X-ray pulsar 1E 1048.1-5937 performed with the XMMNewton satellite. The phase averaged spectrum of 1E 1048.1-5937 is well described by the sum of a power law with photon index $\sim 2.9$ and a blackbody with temperature $\sim 0.6 \mathrm{keV}$, without evidence for significant absorption or emission lines. The above spectral parameters do not vary during the phases corresponding to the broad pulse, while the off pulse emission shows a different spectrum characterized by a soft excess at energies below $\sim 1.5 \mathrm{keV}$. The XMM-Newton observation and a re-analysis of archival BeppoSAX data show that the spectral parameters and flux of 1E 1048.1-5937 did not change significantly during observations spanning the last four years. All the data are consistent with a $2-10 \mathrm{keV}$ luminosity varying in the range $\sim(5-7) \times 10^{33} \mathrm{erg} \mathrm{s}^{-1}$ (for a distance of $3 \mathrm{kpc}$ ).
\end{abstract}

Key words. stars: individual: 1E 1048.1-5937 - X-rays: stars

\section{Introduction}

The pulsar 1E 1048.1-5937 is a persistent source of X-rays with flux of $\sim 10^{-11} \mathrm{erg} \mathrm{cm}^{-2} \mathrm{~s}^{-1}$ and a significant modulation at a period of $\sim 6.45 \mathrm{~s}$ (Seward et al. 1986; Mereghetti 1995; Corbet \& Mihara 1997; Oosterbroek et al. 1998). It belongs to the small group of Anomalous X-ray Pulsars (AXPs, Mereghetti \& Stella 1995; van Paradijs et al. 1995). The nature of these pulsars, that show properties clearly different from those of the $\sim 90$ $\mathrm{X}$-ray pulsars powered by accretion from high mass companion stars, is currently unclear (see Mereghetti 2001, for a review). The lack of evidence for bright optical counterparts led to models for AXPs involving isolated neutron stars, but their typical values of $P$ and $\dot{P}$ yield rotational energy losses inadequate to power the observed luminosity. Although low mass companions cannot be completely ruled out (Mereghetti et al. 1998), it has been proposed that the AXPs consist of isolated neutron stars accreting from residual disks (van Paradijs et al. 1995; Ghosh et al. 1997; Chatterjee et al. 2000). Another class of models is based on isolated, strongly magnetized $\left(B>10^{14} \mathrm{G}\right)$ neutron stars, or "Magnetars" (Thompson \& Duncan 1993, 1996; Heyl \& Hernquist 1997), powered by the decay of their magnetic energy.

Send offprint requests to: A. Tiengo,

e-mail: tiengo@ifctr.mi.cnr.it
Here we report on a new observation of $1 \mathrm{E}$ 1048.15937 performed with the XMM-Newton satellite. The new data provide an accurate measurement of the flux, phase resolved spectroscopy and a more precise localization of this pulsar. We have also reanalyzed the BeppoSAX data originally reported by Oosterbroek et al. (1998) in order to allow a more accurate comparison with the new observation.

\section{Observations and data analysis}

An observation of 1E 1048.1-5937 was carried out with the XMM-Newton satellite (Jansen et al. 2001) on 2000 December 28. Here we concentrate on the data obtained with the European Photon Imaging Camera (EPIC, Turner et al. 2001; Strüder et al. 2001). The short duration of this observation $(\sim 8 \mathrm{ks})$, prevented a significant detection of 1E 1048.1-5937 in the Reflection Grating Spectrometer (RGS).

EPIC consists of two MOS CCD detectors and a PN CCD instrument. In total, the 3 EPICs provide $>2500 \mathrm{~cm}^{2}$ of collecting area at $1.5 \mathrm{keV}$. The mirror system offers an on-axis FWHM angular resolution of $4-5^{\prime \prime}$ and a field of view (FOV) of $30^{\prime}$ diameter.

During this observation the medium filter was used. The MOS cameras were operated in Small Window mode to limit source photon pile-up effects and to improve the 
Table 1. Summary of the spectral results ${ }^{a}$.

\begin{tabular}{|c|c|c|c|c|c|c|}
\hline Obs. & $\begin{array}{c}\text { Pow Law } \\
\text { photon index }\end{array}$ & $\begin{array}{l}\text { Absorption } \\
10^{22} \mathrm{~cm}^{-2}\end{array}$ & $\begin{array}{c}k T \\
(\mathrm{keV})\end{array}$ & $\chi^{2} /$ d.o.f. & $\begin{array}{c}2-10 \mathrm{keV} \text { Flux } \\
\text { absorbed }^{d}\end{array}$ & $\begin{array}{c}2-10 \mathrm{keV} \text { Flux } \\
\text { unabsorbed }\left(\mathrm{erg} \mathrm{cm}^{-2} \mathrm{~s}^{-1}\right)\end{array}$ \\
\hline $\mathrm{PN}$ & $2.7 \pm 0.3$ & $0.9 \pm 0.1$ & $0.61 \pm 0.04$ & $132.1 / 129$ & $3.8 \pm 0.4 \times 10^{-12}$ & $4.3 \times 10^{-12}$ \\
\hline MOS1 & $3.1 \pm 0.4$ & $1.2 \pm$ & $0.63 \pm 0.07$ & $94.7 / 100$ & $4.2 \pm 0.5 \times 10^{-12}$ & $5.1 \times 10^{-12}$ \\
\hline MOS2 & $3.3 \pm 0.6$ & $1.1 \pm 0.2$ & $0.71 \pm 0.07$ & $100.2 / 96$ & $4.0 \pm 0.5 \times 10^{-12}$ & $4.8 \times 10^{-12}$ \\
\hline $\mathrm{EPIC}^{b}$ & $2.9 \pm 0.2$ & $1.04 \pm 0.08$ & $0.63 \pm 0.03$ & $340.5 / 332$ & $4.1 \pm 0.4 \times 10^{-12}$ & $4.9 \times 10^{-12}$ \\
\hline BeppoSAX $X^{c}$ & $3.3 \pm 0.4$ & $1.2 \pm 0.3$ & $0.62 \pm 0.04$ & $185.8 / 180$ & $5.9 \pm 0.5 \times 10^{-12}$ & $7.1 \times 10^{-12}$ \\
\hline
\end{tabular}

${ }^{a}$ Errors are at the $90 \%$ c.l. for a single interesting parameter.

${ }^{b} \mathrm{EPIC}=\mathrm{PN}+\mathrm{MOS} 1+\mathrm{MOS} 2$.

${ }^{c}$ Reanalysis of the observation reported by Oosterbroek et al. (1988).

${ }^{d}$ The errors on the flux values are dominated by the systematic uncertainties (statistical errors are of the order a few percent).

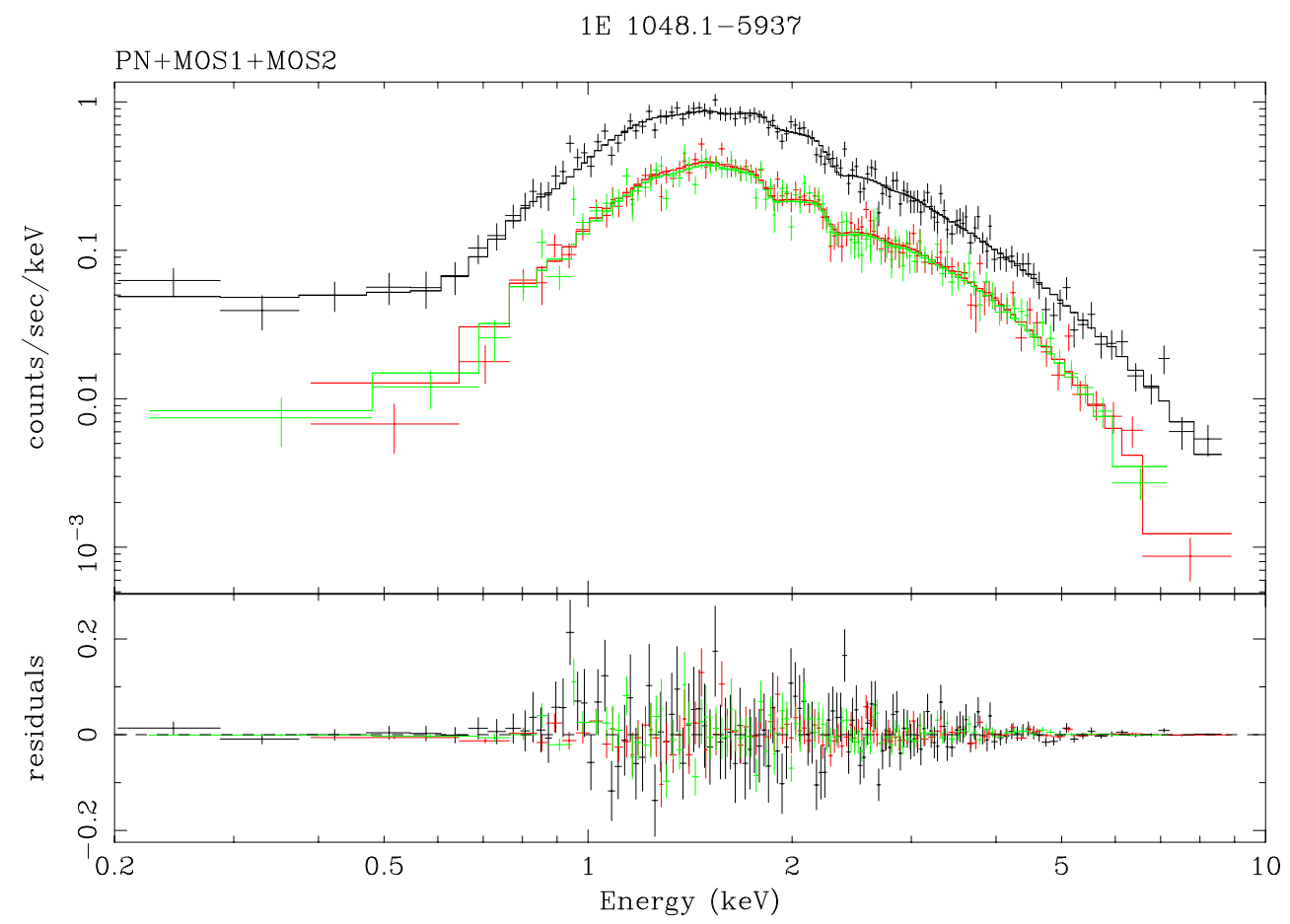

Fig. 1. EPIC spectrum of 1E 1048.1-5937 fitted with the sum of a power law and a blackbody (see the EPIC parameters in Table 1). The upper spectrum is from the PN camera.

time resolution. Due to its shorter frame integration time $(73 \mathrm{~ms})$, the PN could operate in the standard Full Frame mode. The net exposure time was $7135 \mathrm{~s}$ in each MOS camera and $4744 \mathrm{~s}$ in the $\mathrm{PN}$.

The EPIC data reduction was performed using version v5.0 of the XMM-Newton Science Analysis System. An additional data cleaning was required for MOS2 data in order to remove some residual low energy electronic noise.

\subsection{Spectral analysis}

The target was clearly detected in the three EPIC cameras at coordinates $\mathrm{RA}=10^{\mathrm{h}} 50^{\mathrm{m}} 06.3^{\mathrm{s}}$ Dec $=-59^{\circ} 53^{\prime} 17^{\prime \prime}$ (J2000). Based on the current performance of the XMMNewton satellite attitude reconstruction we conservatively estimate an uncertainty of $\sim 4^{\prime \prime}$ on this position that is consistent with previous, less accurate measurements (Mereghetti et al. 1992).
The spectral analysis with the PN camera was based on counts extracted from a $100^{\prime \prime} \times 100^{\prime \prime}$ box centered at the source position. This box was inside a single CCD chip. For the background extraction we tried several sourcefree regions close to the target, without finding significant differences in the results. All the spectra were extracted in the nominal $0.2-10 \mathrm{keV}$ range and rebinned so that at least 20 counts were included in each energy bin and the channels did not oversample the PN energy resolution.

We first analyzed spectra based on both single and double events. This gives good statistics without significantly degrading the spectral performances.

Single component models, i.e. power law, thermal bremsstrahlung and blackbody, gave unacceptable results. A very good fit was obtained with the "canonical" AXP model based on the sum of a blackbody and a power law. The best fit parameters were a blackbody temperature $k T \sim 0.6 \mathrm{keV}$ and a power law photon index $\alpha_{\mathrm{ph}} \sim 2.7$. 


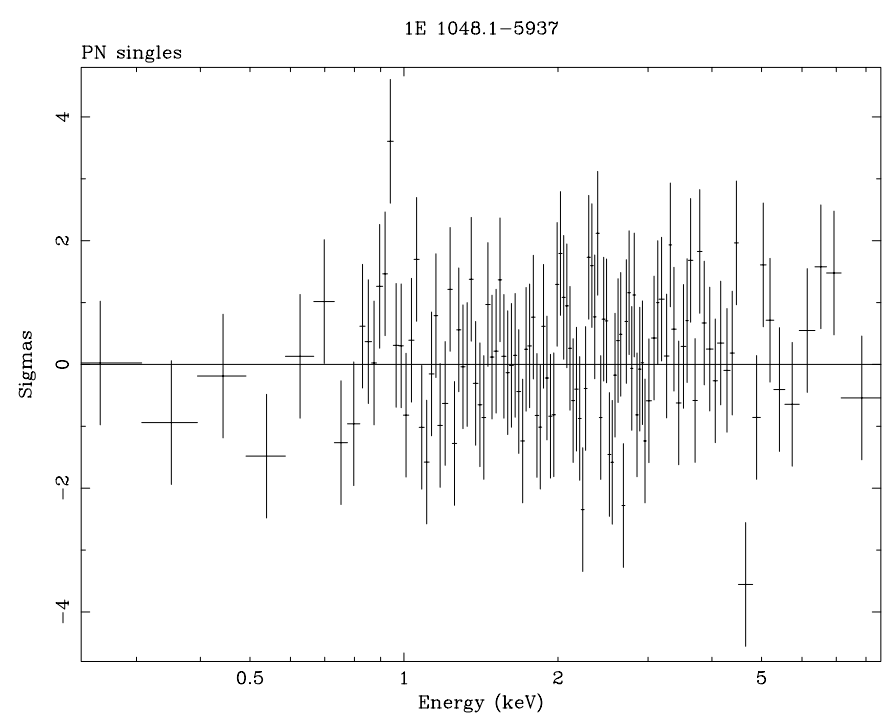

Fig. 2. Residuals of the PN single events spectrum.

The two MOS spectra were based on all the counts of the small CCD windows $\left(100^{\prime \prime} \times 100^{\prime \prime}\right)$ used in this observation. The background could only be extracted from the peripheral CCD chips, excluding small regions around sources and correcting the intensity for the vignetting effect that is significant in this region more than $5^{\prime}$ offset from the telescope axis. We only considered the $0.2-10 \mathrm{keV}$ energy range and applied the same rebinning as in the PN case. For both MOS spectra a combination of blackbody and power law gave the best fit result, with spectral parameters consistent with those obtained with the PN camera.

We therefore proceeded to a joint spectral analysis of the three data sets, allowing only the relative normalization to vary. The results are reported in Table 1 for the blackbody plus power law model and the corresponding spectrum is shown in Fig. 1.

The observed flux in the $2-10 \mathrm{keV}$ energy range is $\sim 4.1 \times 10^{-12} \mathrm{erg} \mathrm{cm}^{-2} \mathrm{~s}^{-1}$ in the two MOS cameras. The value obtained with the PN is about $10 \%$ smaller. Part of the discrepancy can be explained by the fact that in our analysis the PN "Out of Time" events are excluded. They are estimated to be the $6.2 \%$ of the total flux in Full Frame mode. The remaining discrepancy is within the uncertainties in the relative calibration of the instruments.

The best fit spectral parameters reported in Table 1 are consistent with those derived from previous ASCA observations (Paul et al. 2000), but they show some differences with respect to those observed with BeppoSAX by Oosterbroek et al. (1998), who found a harder powerlaw $\left(\alpha_{\mathrm{ph}} \sim 2.5\right)$ and a smaller absorption $\left(N_{\mathrm{H}} \sim 0.45 \times\right.$ $10^{22} \mathrm{~cm}^{-2}$ ). In order to check if this discrepancy is really due to a spectral variation we present a reanalysis of the BeppoSAX data in Sect. 2.4.

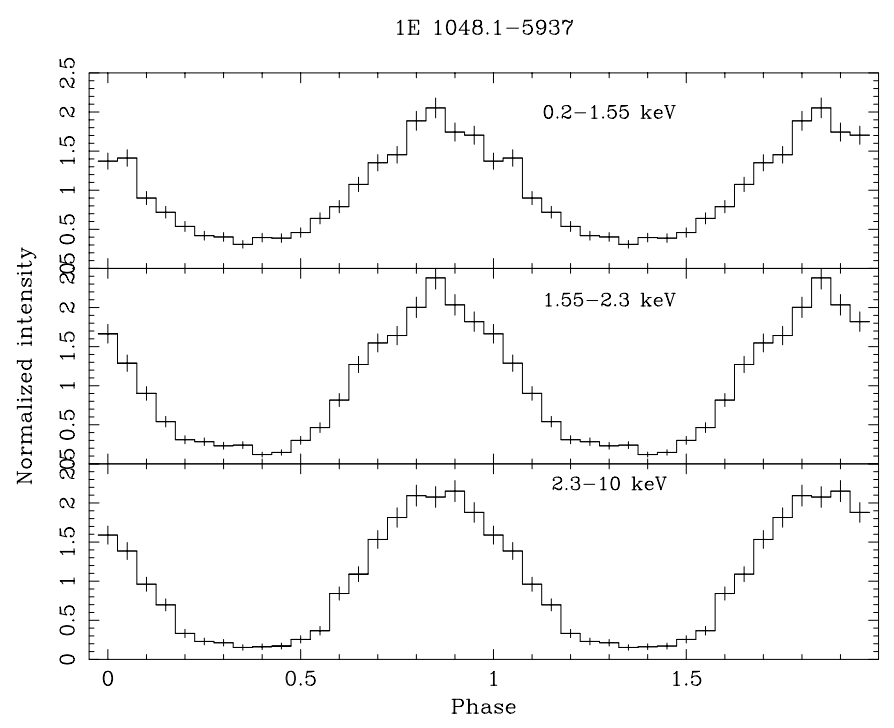

Fig. 3. EPIC PN light curves of 1E 1048.1-5937 folded at the best period for different energy ranges.

\subsection{Search for spectral features}

To fully exploit the energy resolution provided by the EPIC CCD detectors, we performed a spectral analysis by selecting only single events. Spectra based on single events, i.e. those for which the collected charge is not split between adjacent CCD pixels, provide the best energy resolution, at the expenses of a slightly reduced efficiency.

To extract and rebin source and background spectra from the PN camera we followed the same procedure described in the previous section. The residuals of the best fit spectrum (power law plus blackbody) are shown in Fig. 2. Possible features are seen at energies of $\sim 0.95 \mathrm{keV}$ and in the region between $\sim 4$ and $5 \mathrm{keV}$.

Although the highest point in the low energy feature is at $\sim 3.5 \sigma$ from the best fit model, the fact that its width is smaller than the instrumental resolution at this energy $(F W H M \sim 60-70 \mathrm{eV})$ suggests that it is most likely an artifact.

The absorption at high energy has a width consistent with the instrumental resolution. It can be fit with a Gaussian line centered at $E=4.71 \pm 0.04 \mathrm{keV}$ and with $\sigma=45 \mathrm{eV}$.

None of these two possible features is confirmed by the MOS spectra (an excess is seen in the residuals at $\sim 0.9 \mathrm{keV}$ only in the MOS2 at $\sim 2 \sigma$ ).

\subsection{Timing analysis and phase resolved spectroscopy}

The timing analysis was done by correcting the time of arrival of the counts to the Solar System barycenter and then folding them to several trial periods around the expected value. The best periods obtained for the PN and MOS data were respectively $P=6.4526 \pm 0.0001$ and $P=6.4525 \pm 0.0001$.

The PN folded light curve is shown in Fig. 3 for different energy ranges. The pulse profile is characterized 


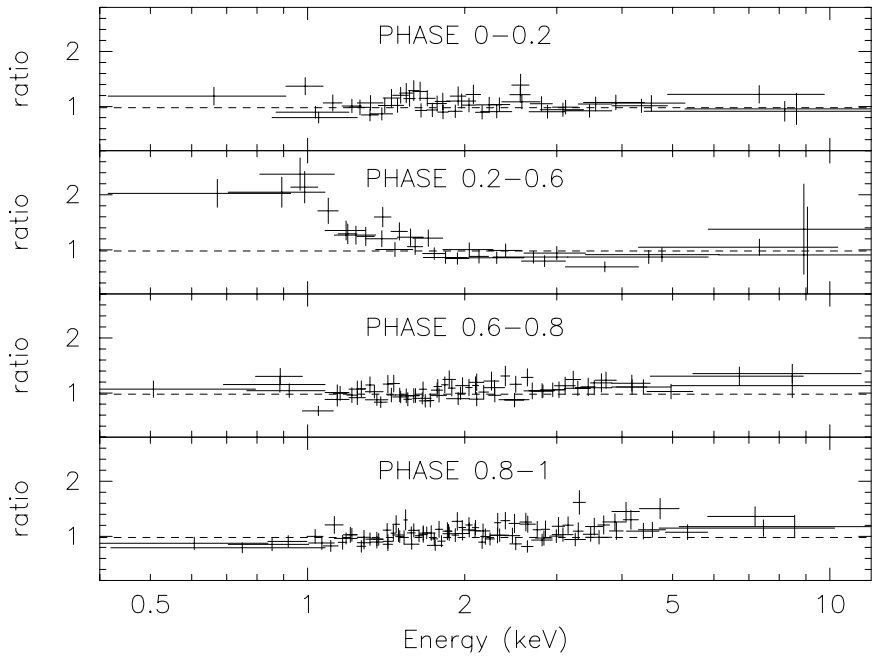

Fig. 4. Results of the phase resolved spectroscopy. The figures show the ratios between the source spectra in the indicated phase intervals and the average spectrum.

by a broad, nearly sinusoidal profile at all energies, as already noted in previous observations with other satellites. The level of modulation was derived by fitting a constant plus a sinusoid to the normalized pulse profiles, yielding a pulsed fraction of $\sim 72 \%$ below $1.5 \mathrm{keV}$ and $\sim 94 \%$ at higher energies.

To search for possible spectral variations as a function of the spin phase, we divided the data in four phase intervals corresponding to the off-pulse $(0.2-0.6)$, rising $(0.6-0.8)$, top $(0.8-1)$, and declining $(0-0.2)$ parts of the light curve. The spectra of the four phase intervals were fitted with the power law plus blackbody model, allowing only the overall normalization to vary and keeping all the other parameters fixed at the best fit values of Table 1. The resulting residuals, see Fig. 4, clearly illustrate that the average spectral parameters provide a satisfactory fit to the three phase intervals covering the rise, peak and decay of the pulse. On the other hand, the spectrum during the phase interval $0.2-0.6$ (corresponding to the minimum in the folded light curve) presents a significant excess below $\sim 1.5 \mathrm{keV}$.

Despite the limited counting statistics, we attempted to model the spectral shape in the pulse minimum. A single power law provides an acceptable fit with parameters $\alpha_{\mathrm{ph}} \sim 3.0$ and $N_{\mathrm{H}} \sim 9 \times 10^{21} \mathrm{~cm}^{-2}$. Keeping the absorption fixed at the phase averaged value of $10^{22} \mathrm{~cm}^{-2}$, we obtain $\alpha_{\mathrm{ph}}=3.2 \pm 0.1$.

\subsection{Reanalysis of the BeppoSAX data}

We reanalyzed the BeppoSAX data of the May 1997 observation already reported by Oosterbroek et al. (1998). The main difference with respect to the work of these authors is our estimate of the background spectrum from a region of the field close to the target. Since 1E 1048.15937 lies at low galactic latitude, where significant diffuse
$\mathrm{X}$-ray emission from the Carina nebula is present, the use of standard background files from BeppoSAX observations of extragalactic empty fields underestimates the background level (particularly at energies below $\sim 3 \mathrm{keV}$ ).

Our extraction regions for the MECS (2-10 keV) were a circle of radius $4^{\prime}$ for the source and a circular corona from $280^{\prime \prime}$ to $560^{\prime \prime}$ for the background. The corresponding LECS (0.5-10 keV) values were $8^{\prime}, 520^{\prime \prime}$ and $960^{\prime \prime}$. The best fit results to the joint LECS+MECS spectra for the power law plus blackbody model are given in Table 1. Both the absorption and the power law photon index are different from those reported by Oosterbroek et al. (1998) and consistent with the EPIC values. Thus we conclude that there is no evidence for a variation in the source spectrum between the two observations. The unabsorbed flux corresponding to the best fit BeppoSAX parameters is $7.1 \times 10^{-12} \mathrm{erg} \mathrm{cm}^{-2} \mathrm{~s}^{-1}(2-10 \mathrm{keV})$, about $45 \%$ higher than that observed with XMM-Newton.

\section{Discussion}

The XMM-Newton observation of 1E 1048.1-5937 provides the first clear evidence for a spectral variation as a function of the pulse phase in this source. The spectrum shows a "soft excess" (with respect to the phase averaged spectrum) during the pulse minimum, while no significant variations occur along the pulse rise and decay. This behavior is different from that of other AXPs which show energy dependent pulse shapes like 1RXS J170849-4009 (Israel et al. 2001; Gavriil \& Kaspi 2001) and 4U 0142+61 (Israel et al. 1999).

Although the spectra of all AXPs are well described by the sum of a power law and a blackbody, there are no compelling reasons to attribute this spectral shape to the presence of two physically distinct emitting components (some suggestions in this sense were made, e.g., by Ghosh et al. 1997). In fact, as noted by Özel et al. (2001), the contribution of the blackbody component to the total flux is a strong function of the energy, while the pulsed fraction at different energies does not change significantly. In the case of two physically distinct spectral components this would require an ad hoc coupling in the pulse profiles of the blackbody and power law components.

The fact that the pulsed emission from 1E 1048.15937 can be fit by the same spectral shape at all the phases also suggests that it originates from a single physical component, with a spectrum more complex than a pure blackbody. Theoretical AXPs spectra have been recently computed by several authors in the context of models of thermal emission from strongly magnetized neutron stars. These models predict modifications to pure blackbody-like spectra, in the form of hard power-law tails (Özel 2001; Perna et al. 2001) and/or narrow spectral features (Zane et al. 2001). Further observations with better statistics are clearly required to confirm the possible features in the spectrum of 1E 1048.1-5937 reported in Sect. 2.2.

The period value found in the XMM-Newton observation is consistent with the overall spin-down trend at an 
average $\dot{P} \sim 1.6 \times 10^{-11} \mathrm{~s} \mathrm{~s}^{-1}$ that $1 \mathrm{E} 1048.1-5937$ has maintained in the last few years. Large variations in the average spin-down rate were detected in the past. In particular, between June 1992 and March 1994 (Mereghetti 1995; Corbet \& Mihara 1997), $\dot{P} \sim 3.3 \times 10^{-11} \mathrm{~s} \mathrm{~s}^{-1}$ was about a factor 2 higher than that measured earlier and after 1996. More recent observations have clearly shown that the level of rotational irregularities in 1E 1048.1-5937 is higher than in other sources of this class (Paul et al. 2000; Baykal et al. 2000, 2001; Kaspi et al. 1999, 2001; Gavriil \& Kaspi 2001).

In principle, the study of the spin-down evolution in AXPs could discriminate between accretion-based and magnetar models. If AXPs are powered by accretion (either from a companion star or from a residual disk) one would expect significant $\dot{P}$ fluctuations superimposed on the long term spin-down trend, possibly correlated with luminosity variations, due to irregularities in the accretion flow. On the other hand, the period evolution of a magnetar should be much more regular, with the possible exception of "glitches" as observed in young radio pulsars.

It is unclear whether the spin-down variations seen in 1E 1048.1-5937 are related to changes in its $\mathrm{X}$-ray flux, particularly for observations carried out before 1995 . Oosterbroek et al. (1998) compared the flux of 1E 1048.15937 measured with BeppoSAX to all the values obtained in the previous years with the Einstein, EXOSAT, ROSAT and ASCA satellites. Although the flux values span almost an order of magnitude, the systematic uncertainties are difficult to evaluate, since some of these measurements referred to different energy ranges. Furthermore, the data from non-imaging detectors might be contaminated by the presence of the strongly variable source Eta Carinae within the field of view.

By means of a systematic program aimed at phasecoherent timing of 1E 1048.1-5937 with the RossiXTE satellite, Kaspi et al. (1999) showed the presence of significant $\dot{P}$ changes between 1996 and 2000. Their ephemerids are shown by the solid curves in Fig. 5. Unfortunately, due to difficulties in the background subtraction, the RossiXTE non-imaging observations can measure only the intensity of the pulsed flux, which was found consistent with a constant value. Our XMM-Newton and BeppoSAX results, compared with the ASCA ones (Paul et al. 2000), show that all the measurements obtained since 1994 with imaging detectors are consistent with an unabsorbed $2-10 \mathrm{keV}$ flux within the range $\sim(5-7) \times$ $10^{-12} \mathrm{erg} \mathrm{cm}^{-2} \mathrm{~s}^{-1}$.

Our period measurement is inconsistent with an extrapolation of the phase-coherent solution reported by Kaspi et al. (2001). This is a further demonstration of the high level of timing noise present in 1E 1048.1-5937.

\section{Conclusions}

The large collecting area of the XMM-Newton telescope has provided the first significant evidence for spectral variations as a function of the spin phase in 1E 1048.1-5937.

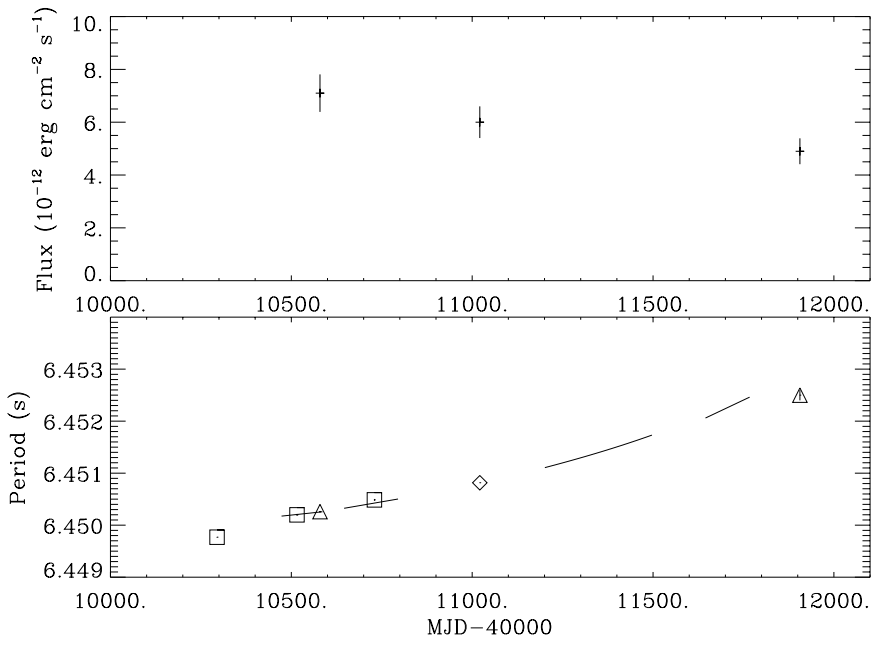

Fig. 5. Flux and spin period evolution of 1E 1048.1-5937 during the years $1996-2000$. The top panel reports the $2-10 \mathrm{keV}$ flux corrected for absorption as measured with BeppoSAX, ASCA and $X M M$-Newton. The different symbols in the bottom panel are as follows. Triangles: BeppoSAX and XMM-Newton (this work); squares: RossiXTE (Mereghetti et al. 1998; Baykal et al. 2000); diamonds: ASCA (Paul et al. 2000); solid lines: RossiXTE (Kaspi et al. 2001).

Contrary to other AXPs, the spectral shape changes only in the off-pulse emission, while no significant variations are seen during the broad pulse.

No convincing evidence was found for narrow spectral features. Longer XMM-Newton observations (also exploiting the RGS instrument) and improved calibrations of the instruments are required to verify (or not) the structures marginally detected in the EPIC PN camera.

The XMM-Newton observation, as well as our reanalysis of the archival BeppoSAX data, show that the spectral parameters and flux of 1E 1048.1-5937 did not change significantly during observations spanning the last four years. The fact that all the observations of good quality obtained with imaging detectors and covering energies up to $\sim 10 \mathrm{keV}$ are consistent with a $2-10 \mathrm{keV}$ luminosity varying in the range $\sim(5-7) \times 10^{33} \mathrm{erg} \mathrm{s}^{-1}$ (for $d=3 \mathrm{kpc}$ ), casts some doubts on previous reports of variability up to a factor 10. It is unclear whether the large timing noise of $1 \mathrm{E} 1048.1-5937$ can be ascribed to variations in the torque exerted on the neutron star by such relatively small fluctuations in the mass accretion rate.

Finally, we have provided a reduction of a factor $\sim 15$ in the area of the error box of 1E 1048.1-5937. This will undoubtedly help in the search for an optical counterpart of this intriguing $\mathrm{X}$-ray source.

Acknowledgements. E. Gö. and R. St. acknowledge the support through a grant by DLR (Verbundforschung).

\section{References}

Baykal, A., Strohmayer, T., Swank, J. H., Alpar, M. A., \& Stark, M. J. 2000, MNRAS, 319, 205 
Chatterjee, P., Hernquist, L., \& Narayan, R. 2000, ApJ, 534, 373

Corbet, R. H. D., \& Mihara, T. 1997, ApJ, 475, L127

Gavriil, F. P., \& Kaspi, V. M. 2001, ApJ, in press [astro-ph/0107422]

Ghosh, P., Angelini, L., \& White, N. E. 1997, ApJ, 478, 713

Israel, G. L., Oosterbroek, T., Angelini, L., et al. 1999, A\&A, 346, 929

Israel, G. L., Oosterbroek, T., Stella, L., et al. 2001, ApJ, 560, L65

Jansen, F., Lumb, D., Altieri, B., et al. 2001, A\&A, 365, L1

Kaspi, V. M., Chakrabarty, D., \& Steinberger, J. 1999, ApJ, 525, L33

Kaspi, V. M., Lackey, J. R., \& Chakrabarty, D. 2000, ApJ, 537, L31

Kaspi, V. M., Gavriil, F. P., Chakrabarty, D., Lackey, J. R., \& Muno, M. P. 2001, ApJ, in press [astro-ph/0011368]

Mereghetti, S. 1995, ApJ, 455, 598

Mereghetti, S. 2001, in The Neutron Star Black Hole Connection, ed. C. Kouveliotou, J. van Paradijs, \& J. Ventura, NATO ASI Ser. (Dordrecht Kluwer), in press [astro-ph/9911252]

Mereghetti, S., \& Stella, L. 1995, ApJ, 442, L17
Mereghetti, S., Caraveo, P., \& Bignami, G. F. 1992, A\&A, 263, 172

Mereghetti, S., Israel, G. L., \& Stella, L. 1998, MNRAS, 296, 689

Oosterbroek, T., Parmar, A. N., Mereghetti, S., \& Israel, G. L. 1998, A\&A, 334, 925

Özel, F., Psaltis, D., \& Kaspi, V. M. 2001, ApJ, submitted [astro-ph/0105372]

Özel, F. 2001, ApJ, submitted [astro-ph/0103227]

Paul, B., Kawasaki, M., Dotani, T., \& Nagase, F. 2000, ApJ, 537,319

Perna, R., Heyl, J. S., Hernquist, L. E., Juett, A. M., \& Chakrabarty, D. 2001, ApJ, 557, 18

Seward, F., Charles, P. A., \& Smale, A. P. 1986, ApJ, 305, 814

Strüder, L., Briel, U., Dennerl, K., et al. 2001, A\&A, 365, L18

Thompson, C., \& Duncan, R. C. 1993, ApJ, 408, 194

Thompson, C., \& Duncan, R. C. 1996, ApJ, 473, 322

Turner, M. J. L., Abbey, A., Arnaud, M., et al. 2001, A\&A, 365, L27

van Paradijs, J., Taam, R. E., \& van den Heuvel, E. P. J. 1995, A\&A, 299, L41

Zane, S., Turolla, R., Stella, L., \& Treves, A. 2001, ApJ, in press [astro-ph/0103316] 